\author{
G.A. Yessenbayeva ${ }^{1, *}$, G.A. Yessenbayeva ${ }^{2}$, A.T. Kasimov ${ }^{3}$, N.K. Syzdykova ${ }^{1}$ \\ ${ }^{1}$ Karagandy university of the name of academician E.A. Buketov, Karaganda, Kazakhstan; \\ ${ }^{2}$ Karaganda University of Kazpotrebsoyuz, Karaganda, Kazakhstan; \\ ${ }^{3}$ Karaganda Technical University, Karaganda, Kazakhstan \\ (E-mail: work.ksu21@mail.ru, esenbaeva_keu@mail.ru,kasimov5301@mail.ru,s_nazym_1807@mail.ru)
}

\title{
On the boundedness of the partial sums operator for the Fourier series in the function classes families associated with harmonic intervals
}

\begin{abstract}
The article is devoted to the study of some data from the theory of functions approximation by trigonometric polynomials with a spectrum from special sets called harmonic intervals. Due to the limited perception range of devices, the perception range of the senses of the person himself, when studying a mathematical model it is often enough to find an approximation of the object so that the error (noise, interference, distortion) is outside the interval of perception. Harmonic intervals model problems of this kind to some extent. In the article the main components of the approximation theory of functions by trigonometric polynomials with a spectrum from harmonic intervals are presented, the theorem on estimating the best approximation of a function by trigonometric polynomials through the best approximations of a function by trigonometric polynomials with a spectrum from harmonic intervals is proved. Theorems on the boundedness of the partial sums operator for the Fourier series in the function classes families associated with harmonic intervals are considered; such a theorem for the Lorentz space is generalized and proved. The article is mainly aimed at scientific researchers dealing with practical applications of the approximation theory of functions by trigonometric polynomials with a spectrum from special sets.
\end{abstract}

Keywords: harmonic interval, trigonometric polynomials with a spectrum from harmonic intervals, best approximation of a function by trigonometric polynomials, partial sums operator of the Fourier series for a given function, interpolation theorem.

\section{Introduction}

In approximation theory one of the most relevant problems is the approximation of periodic functions by polynomials with a spectrum from special families of sets. Here we note the works of K.I. Babenko, S.A. Telyakovsky, V.N. Temlyakov [1] and others in the case when the spectrum is a hyperbolic cross; the works of V.I. Yudin, M.I. Dyachenko [2] in the case when the spectrum is a ball, etc.

In the study of many applied problems the question of approximating the mathematical model of the object under study naturally arises. However, due to the limited range of perception («window of perception») of devices, the range of perception of the human senses, when studying a mathematical model it is often enough to find an approximation of the object so that the error (noise, interference, distortion) is outside the interval («window») of perception.

In this paper we consider approximations of functions by trigonometric polynomials with a spectrum from harmonic intervals, which to some extent model problems of this kind.

Note that harmonic intervals are some fractal self-similar sets, the concept of which was introduced by E.D. Nursultanov in [3-5] and, as it turned out, harmonic intervals have an important role in harmonic analysis. Thus, in the works of N.T. Tleukhanova, K.S. Saydakhmetov, D.S. Karimov, such objects as harmonic segments and harmonic intervals were essentially used.

In the studying the problem of the boundedness of the partial sums operator for the Fourier series in the function classes families associated with the best approximations over harmonic intervals the method of real interpolation is used. Among the works devoted to the properties of interpolation spaces, as well as to the methods of interpolation, one should note the works of Y. Berg and J. Lefstrom [6], S.G. Krein, Yu.I. Petunin, E.M. Semenov, Yu.A. Brudny [7], [8], H. Tribel [9], [10].

\footnotetext{
${ }^{*}$ Corresponding author.

E-mail: work.ksu21@mail.ru
} 


\section{Definitions and auxiliary results}

Let $k, \nu, N \in \mathbb{N}, k<N$. A set of the form

$$
I_{k}^{N}=\bigcup_{\nu=-\infty}^{\infty}([-k, k]+2 \nu N)=\bigcup_{\nu=-\infty}^{\infty}(m+2 \nu N: m \in[-k, k])
$$

is called a harmonic interval in $\mathbb{Z}$.

We denote by $T_{k}^{N}$ the set of trigonometric polynomials of the form

$$
T_{k}^{N}=\left\{\sum_{\nu=-s}^{s} a_{\nu} \cdot e^{i \nu x}: a_{\nu}=0 \text { if } \nu \notin I_{k}^{N}, s \in \mathbb{N}\right\}
$$

The value

$$
E_{k}^{N}(f)_{p}=\inf _{t \in T_{k}^{N}}\|f-t\|_{p}
$$

is called the best approximation over the harmonic interval $I_{k}^{N}$ of the function $f \in L_{p}[0,2 \pi), 1 \leq p \leq \infty$, by trigonometric polynomials from $T_{k}^{N}$ of order less than or equal to $k$.

Let $f \in L_{p}[0,2 \pi), 1 \leq p \leq \infty$. The partial sum of the Fourier series for the function $f$ over the harmonic interval $I_{k}^{N}$ is called the function

$$
S_{k}^{N}(f)=\sum_{\nu \in I_{k}^{N}} a_{\nu} \cdot e^{i \nu x} .
$$

Theorem 1. [11] Let $f \in L_{p}[0,2 \pi), 1<p<\infty, m \in \mathbb{N}$. $S_{m}^{N}(f)$ and $E_{m}^{N}(f)$ are the partial sum of the Fourier series and the best approximation of the function $f$ over the harmonic interval $I_{m}^{N}$ respectively, then we have the following relation

$$
E_{m}^{N}(f)_{p} \sim\left\|f-S_{m}^{N}(f)\right\|_{p} .
$$

Lemma 1. [11] Let $n \in \mathbb{N}, 1 \leq p<q \leq \infty, 1 \leq r \leq \infty$, then

$$
\left\|T_{n}\right\|_{L_{q, r}} \leq C n^{\frac{1}{p}-\frac{1}{q}}\left\|T_{n}\right\|_{L_{p}} .
$$

Let $1 \leq p, q \leq \infty, r>0, f \in L_{p}[0,2 \pi)$. The family of function classes $\left\{B_{p, q . N}^{r}\right\}_{N}$ is defined by the equality

$$
B_{p, q . N}^{r}=\left\{f:\|f\|_{B_{p, q . N}^{r}}<\infty\right\}, N \in \mathbb{N}
$$

where

$$
\|f\|_{B_{p, q . N}^{r}}=\left(\sum_{k=1}^{N} k^{r q-1}\left(E_{k-1}^{N}(f)_{p}\right)^{q}\right)^{\frac{1}{q}} .
$$

Let two families of function classes $\left\{A^{N}\right\}_{N}$ and $\left\{B^{N}\right\}_{N}, N \in \mathbb{N}$, be given. We assume that the ratio

$$
\|f\|_{A^{N}} \sim\|f\|_{B^{N}}
$$

holds if there are parameters $C_{1}, C_{2}$ such that for any $f \in A^{N}$ the following inequality

$$
C_{1}\|f\|_{B^{N}} \leq\|f\|_{A^{N}} \leq C_{2}\|f\|_{B^{N}}
$$

is valid, moreover, the parameters $C_{1}, C_{2}$ do not depend on $f$ and $N$.

Theorem 2. [12] Let $f \in B_{p, q .2^{m}}^{r}, m \in \mathbb{N}$. Then for $1 \leq p, q \leq \infty, r>0$ we have

$$
\|f\|_{B_{p, q \cdot 2^{m}}^{r}} \sim\left(\sum_{k=1}^{m} 2^{r q k}\left(E_{2^{k}-1}^{2^{m}}(f)_{p}\right)^{q}\right)^{\frac{1}{q}} .
$$

Theorem 3. [12] Let $m \in \mathbb{N}, 1 \leq p, p_{0}, p_{1} \leq \infty, 0<\theta<1, r_{0}>0, r_{1}>0, r_{0} \neq r_{1}, \frac{1}{p}=\frac{1-\theta}{p_{0}}+\frac{\theta}{p_{1}}$, $r=(1-\theta) \cdot r_{0}+\theta \cdot r_{1}$, then

$$
\left(B_{p_{0}, p_{0}, 2^{m}}^{r_{0}} ; B_{p_{1}, p_{1}, 2^{m}}^{r_{1}}\right)_{\theta, p}=B_{p, p, 2^{m}}^{r} .
$$


Estimation by the best approximations over harmonic intervals

Theorem 4. Let $f \in L_{p}[0,2 \pi), 1<p<\infty, n \in \mathbb{N}$. $\sum_{\nu \in \mathbb{Z}} a_{\nu} \cdot e^{i \nu x}$ is the trigonometric Fourier series of the function $f$, then the following inequality holds

$$
E_{n}(f)_{p} \leq \sum_{j=1}^{\infty} E_{\left(2^{j}-1\right) \cdot n}^{2^{j} \cdot n}(f)_{p}
$$

Proof. By Lemma 9.3 [13] we have

$$
E_{n}(f)_{p} \sim\left\|f-S_{n}(f)\right\|_{p},
$$

when $1<p<\infty$ or

$$
E_{n}(f)_{p} \sim\left\|\sum_{\nu \in Z \backslash[-n, n]} a_{\nu} \cdot e^{i \nu x}\right\| .
$$

By entering the notation of harmonic intervals in $\mathbb{Z}$

$$
\begin{gathered}
V_{j}=\bigcup_{m=-\infty}^{\infty}\left\{\left[\left(2^{j}-1\right) n ;\left(2^{j}+1\right) n\right]+2^{j+1} m n\right\}= \\
=\bigcup_{m=-\infty}^{\infty}\left\{[-n ; n]+2^{j} n(2 m+1)\right\}, \quad j=1,2, \ldots,
\end{gathered}
$$

we obtain

$$
\mathbb{Z} \backslash[-n ; n]=\bigcup_{j=1}^{\infty} V_{j} .
$$

Then from (2) we get the relation in this form

$$
E_{n}(f)_{p} \sim\left\|\sum_{j=1}^{\infty} \sum_{\nu \in V_{j}} a_{\nu} \cdot e^{i \nu x}\right\|_{p}=\left\|\sum_{j=1}^{\infty} \sum_{m=-\infty}^{\infty} \sum_{s=n\left[2^{j}(2 m+1)-1\right]}^{n\left[2^{j}(2 m+1)+1\right]} a_{s} \cdot e^{i s x}\right\|_{p} .
$$

We denote by $W_{j}, j=1,2, \ldots$, the following sets

$$
W_{j}=\mathbb{Z} \backslash V_{j},
$$

where

$$
W_{j}=\bigcup_{m=-\infty}^{\infty}\left\{\left[-\left(2^{j}-1\right) n ;\left(2^{j}-1\right) n\right]+2^{j+1} m n\right\}
$$

or

$$
W_{j}=I_{\left(2^{j}-1\right) n}^{2^{j} n} .
$$

We note that the sets $W_{j}, j=1,2, \ldots$ are also harmonic intervals in $\mathbb{Z}$ as complements of the harmonic intervals $V_{j}, j=1,2, \ldots$ in $\mathbb{Z}$. Then, according to Theorem 1 , using (4), from (3) we obtain the required inequality

$$
\begin{gathered}
E_{n}(f)_{p} \sim\left\|\sum_{j=1}^{\infty} \sum_{\nu \in \mathbb{Z} \backslash W_{j}} a_{\nu} \cdot e^{i \nu x}\right\|_{p}=\left\|\sum_{j=1}^{\infty}\left(f-S_{W_{j}}(f)\right)\right\|_{p} \leq \\
\leq \sum_{j=1}^{\infty}\left\|\left(f-S_{W_{j}}(f)\right)\right\|_{p} \sim \sum_{j=1}^{\infty} E_{W_{j}}(f)_{p}, \\
E_{n}(f)_{p} \leq \sum_{j=1}^{\infty} E_{\left(2^{j}-1\right) \cdot n}^{2^{j} \cdot n}(f)_{p} .
\end{gathered}
$$

The theorem is proved. 
Theorems on the boundedness of the partial sums operator for the Fourier series of a function $f$ in the function classes families $\left\{B_{p, q . N}^{r}\right\}_{N}$

Theorem 5. [11] Let $N \in \mathbb{N}, 1 \leq p<q \leq \infty, 1 \leq r \leq \infty, \beta>0, \alpha-\beta=\frac{1}{p}-\frac{1}{q}$. $B_{q, r}^{\beta}$ is the Besov space [14], then the partial sums operator for the trigonometric Fourier series of the function $f$

$$
S_{N}(f(x))=\sum_{k=-N}^{N} \widehat{f}(k) e^{i k x}
$$

such that

$$
S_{N}: B_{p, r, N}^{\alpha} \rightarrow B_{q, r}^{\beta}
$$

is bounded, that is, there is the inequality

$$
\left\|S_{N}(f)\right\|_{B_{q, r}^{\beta}} \leq C\|f\|_{B_{p, r, N}^{\alpha}},
$$

where the parameter $C$ do not depend on $f$ and $N$.

Corollary 1. [11] Let $N \in \mathbb{N}, 1 \leq p<q \leq \infty, 1 \leq r \leq \infty, \beta>0, \alpha-\beta=\frac{1}{p}-\frac{1}{q}$, then the partial sums operator for the trigonometric Fourier series of the function $f$

$$
S_{N}: B_{p, r, N}^{\alpha} \rightarrow B_{q, r, N}^{\beta}
$$

is bounded, that is, the following inequality

$$
\left\|S_{N}(f)\right\|_{B_{q, r, N}^{\beta}} \leq C\|f\|_{B_{p, r, N}^{\alpha}}
$$

where the parameter $C$ do not depend on $f$ and $N$.

Theorem 6. [11] Let $m \in \mathbb{N}, 1 \leq p<q \leq \infty, \alpha=\frac{1}{p}-\frac{1}{q}$, then the partial sums operator for the trigonometric Fourier series of the function $f$

$$
S_{2^{m}}: B_{p, q, 2^{m}}^{\alpha} \rightarrow L_{q}
$$

is bounded, that is, we have the following inequality

$$
\left\|S_{2^{m}}(f)\right\|_{L_{q}} \leq C\|f\|_{B_{p, q, 2^{m}}^{\alpha}},
$$

where the parameter $C$ do not depend on $f$ and $m$.

Remark 1. Theorem 6 can be formulated in a more general form.

Let $N \in \mathbb{N}, 1 \leq p<q \leq \infty, \alpha=\frac{1}{p}-\frac{1}{q}$, then the partial sums operator for the trigonometric Fourier series of the function $\mathrm{f}$

$$
S_{N}: B_{p, q, N}^{\alpha} \rightarrow L_{q}
$$

is bounded, that is, there is the inequality of the form

$$
\left\|S_{N}(f)\right\|_{L_{q}} \leq C\|f\|_{B_{p, q, N}^{\alpha}},
$$

where the parameter $C$ do not depend on $f$ and $N$.

We generalize Theorem 6 to Lorentz spaces.

Theorem 7. Let $N \in \mathbb{N}, 1 \leq p<q \leq \infty, 1 \leq r \leq \infty, \alpha=\frac{1}{p}-\frac{1}{q}$, then the partial sums operator for the trigonometric Fourier series of the function $f$

$$
S_{N}: B_{p, r, N}^{\alpha} \rightarrow L_{q, r}
$$

is bounded, that is, this inequality holds

$$
\left\|S_{N}(f)\right\|_{L_{q, r}} \leq C\|f\|_{B_{p, r, N}^{\alpha}},
$$

where the parameter $C$ do not depend on $f$ and $N$. 
Proof. We estimate the norm of the partial sum operator in the Lorentz space

$$
\left\|S_{N}(f)\right\|_{L_{q, r}} \leq \sum_{k=1}^{\left[\log _{2} N\right]}\left\|\sum_{n=-2^{k-1}}^{2^{k}-1} a_{n} e^{i n x}\right\|_{L_{q, r}}=\sum_{k=1}^{\left[\log _{2} N\right]}\left\|\Delta_{k}\left(S_{N}(f)\right)\right\|_{L_{q, r}} .
$$

Applying the inequality of different metrics (1), we transform the relation (5) as follows

$$
\left\|S_{N}(f)\right\|_{L_{q, r}} \leq C \sum_{k=1}^{\left[\log _{2} N\right]} 2^{k\left(\frac{1}{p}-\frac{1}{q}\right)}\left\|\Delta_{k}\left(S_{N}(f)\right)\right\|_{L_{p}}=C \sum_{k=1}^{\left[\log _{2} N\right]} 2^{\alpha k}\left\|\Delta_{k}\left(S_{N}(f)\right)\right\|_{L_{p}} .
$$

Taking into account that $\triangle_{k}\left(S_{N}(f)\right)$ is a partial sum of the function $\sum_{n \in \mathbb{Z} \backslash I_{2^{k-1}-1}^{N}} a_{n} e^{i n x}$ and using the M. Riesz theorem [15], Theorem 1 and Theorem 2, we reduce relation (6) to the form

$$
\begin{gathered}
\left\|S_{N}(f)\right\|_{L_{q, r}} \leq C \sum_{k=1}^{\left[\log _{2} N\right]} 2^{\alpha k}\left\|\Delta_{k}\left(S_{N}(f)\right)\right\|_{L_{p}} \leq \\
\leq C \sum_{k=1}^{\left[\log _{2} N\right]} 2^{\alpha k}\left\|_{n \in \mathbb{Z} \backslash I_{2^{k-1}-1}^{N}} a_{n} e^{i n x}\right\|_{L_{p}}=C \sum_{k=1}^{\left[\log _{2} N\right]} 2^{\alpha k}\left\|f-S_{2^{k-1}-1}^{N}(f)\right\|_{L_{p}} \leq \\
\leq C \sum_{k=1}^{\left[\log _{2} N\right]} 2^{\alpha k} E_{2^{k-1}-1}^{N}(f)_{p}=C \cdot 2^{\alpha} \sum_{k=1}^{\left[\log _{2} N\right]} 2^{\alpha(k-1)} E_{2^{k-1}-1}^{N}(f)_{p} \leq \\
\leq C \sum_{k=1}^{\left[\log _{2} N\right]} 2^{\alpha k} E_{2^{k}-1}^{N}(f)_{p} \sim C\|f\|_{B_{p, 1, N}^{\alpha}} . \\
\Rightarrow\left\|S_{N}(f)\right\|_{L_{q, r}} \leq C\|f\|_{B_{p, 1, N}^{\alpha}} .
\end{gathered}
$$

We take pairs $\left(\alpha_{0}, \alpha_{1}\right),\left(q_{0}, q_{1}\right),\left(r_{0}, r_{1}\right)$ that satisfy the following conditions

$$
\begin{gathered}
\alpha_{0}<\alpha<\alpha_{1}, \quad q_{0}<q<q_{1}, \quad r_{0}<r<r_{1}, \\
\alpha_{0}=\frac{1}{p}-\frac{1}{q_{0}}, \quad \alpha_{1}=\frac{1}{p}-\frac{1}{q_{1}} .
\end{gathered}
$$

Taking into account the relation (7), we obtain the following

$$
\begin{gathered}
S_{N}: B_{p, 1, N}^{\alpha_{0}} \rightarrow L_{q_{0}, r_{0}}, \\
S_{N}: B_{p, 1, N}^{\alpha_{1}} \rightarrow L_{q_{1}, r_{1}}
\end{gathered}
$$

then, by the interpolation theorem [6], we have

$$
S_{N}:\left(B_{p, 1, N}^{\alpha_{0}} ; B_{p, 1, N}^{\alpha_{1}}\right)_{\theta, r} \rightarrow\left(L_{q_{0}, r_{0}} ; L_{q_{1}, r_{1}}\right)_{\theta, r} .
$$

Using Theorem 3, we receive that this relation holds

$$
\left(B_{p, 1, N}^{\alpha_{0}} ; B_{p, 1, N}^{\alpha_{1}}\right)_{\theta, r}=B_{p, r, N}^{\alpha_{\theta}}
$$

where

$$
\alpha_{\theta}=(1-\theta) \cdot \alpha_{0}+\theta \cdot \alpha_{1}, \frac{1}{r}=\frac{1-\theta}{r_{0}}+\frac{\theta}{r_{1}}, 0<\theta<1 .
$$

It follows from the theorem on the interpolation of Lorentz spaces [6] that

$$
\left(L_{q_{0}, r_{0}} ; L_{q_{1}, r_{1}}\right)_{\theta, r}=L_{q_{\theta}, r},
$$


where

$$
\frac{1}{q_{\theta}}=\frac{1-\theta}{q_{0}}+\frac{\theta}{q_{1}}, \frac{1}{r}=\frac{1-\theta}{r_{0}}+\frac{\theta}{r_{1}}
$$

Since there is a dependency

$$
\alpha_{\theta}=(1-\theta) \cdot \alpha_{0}+\theta \cdot \alpha_{1}=(1-\theta)\left(\frac{1}{p}-\frac{1}{q_{0}}\right)+\theta\left(\frac{1}{p}-\frac{1}{q_{1}}\right)=\frac{1}{p}-\frac{1}{q_{\theta}},
$$

then there is $\theta \in(0 ; 1)$ such that

$$
\alpha_{\theta}=\alpha, q_{\theta}=q
$$

As a result, from (8) we obtain the required relation

$$
S_{N}: B_{p, r, N}^{\alpha} \rightarrow L_{q, r},
$$

and

$$
\left\|S_{N}(f)\right\|_{L_{q, r}} \leq C\|f\|_{B_{p, r, N}^{\alpha}} .
$$

where the parameter $C$ do not depend on $f$ and $N$.

The theorem is proved.

Remark 2. In Theorems 5, 7 and Remark 1, the operator $S_{N}(f)$ can be replaced by the operator $S_{n}(f)$, where $0 \leq n \leq N$. Indeed, from M. Riesz's theorem we have

$$
\left\|S_{n}(f)\right\|_{L_{p}} \leq C\left\|S_{N}(f)\right\|_{L_{p}},
$$

where the parameter $C$ do not depend on $f$ and $N$.

\section{References}

1 Темляков В.Н. Дискретизация интегральных норм полиномов с гармониками из гиперболического креста по значениям в точках / В.Н. Темляков // Функциональные пространства, теория приближений и смежные вопросы анализа: сб. ст. к 115-летию со дня рожд. акад. Сергея Михайловича Никольского // Тр. МИАН. - 2021. - 312. - С. 282-293.

2 Теляковский С.А. Очерки развития математики в СССР / С.А. Теляковский. - Киев: Наук. думка, 1983. - C. 237-251.

3 Нурсултанов Е.Д. Сетевые пространства и их приложения к задачам гармонического анализа: дис. ... д-ра ф.-м. наук: 01.01.01 - Вещественный, комплексный и функциональный анализ / Ерлан Даутбекович Нурсултанов. - М., 1999. - С. 171-179.

4 Нурсултанов Е.Д. Сетевые пространства и неравенства Харди-Литтлвуда / Е.Д. Нурсултанов // Мат. сб. - 1998. - Т. 189. - № 3. - С. 83-102.

5 Нурсултанов Е.Д. Сетевые пространства и преобразование Фурье / Е.Д. Нурсултанов // Док. РАН (361). - 1998. - № 5. - С. 597-599.

6 Берг Й. Интерполяционные пространства. Введение / Й. Берг, Й. Лефстрем. - М.: Мир, 1980. $264 \mathrm{c.}$

7 Крейн С.Г. Интерполяция линейных операторов / С.Г. Крейн, Ю.И. Петунин, Е.М. Семенов. - М.: Наука, 1978. - 400 с.

8 Брудный Ю.А. Интерполяция линейных операторов / Ю.А. Брудный, С.Г. Крейн, Е.М. Семенов // Итоги науки и техн. Сер. Мат. анал. - 1986. - Т. 24. - С. 3-163.

9 Трибель Х. Теория интерполяции, функциональные пространства, дифференциальные операторы / Х. Трибель. - М.: Мир, 1980. - 664 с.

10 Трибель Х. Теория функциональных пространств / Х. Трибель. - М.: Мир, 1986. - 448 с.

11 Есенбаева Г.А. О свойствах частичных сумм по гармоническим интервалам и классах функций, связанных с наилучшими приближениями по гармоническим интервалам / Г.А. Есенбаева // Вестн. Караганд. ун-та. Сер. Математика. - 2008. - № 1(49). - С. 29-36. 
12 Есенбаева Г.А. Интерполяция некоторых неравенств и аппроксимация функций тригонометрическими полиномами со спектром из гармонических интервалов / Г.А. Есенбаева, Е.Д. Нурсултанов // Евраз. мат. журн. - 2004. - № 1. - С. 3-20.

13 Смаилов Е.С. Теория приближения и вложения классов функций многих переменных / Е.С. Смаилов, Г.А. Акишев, М.Г. Есмаганбетов. - Караганда: Изд-во КарГУ, 1986. - 87 с.

14 Никольский С.М. Приближение функций многих переменных и теоремы вложения / С.М. Никольский. - М.: Наука, 1977. - 456 с.

15 Бари Н.К. Тригонометрические ряды / Н.К. Бари. - М.: Физматлит, 1961. - 936 с.

\author{
Г.А. Есенбаева ${ }^{1}$, Г.А. Есенбаева ${ }^{2}$, А.Т. Касимов ${ }^{3}$, Н.К. Сыздыкова ${ }^{1}$ \\ ${ }^{1}$ Академик Е.А.Бөкетов атындавы Қараганды университеті, Қараванды, Қазақстан; \\ ${ }^{2}$ Қазтұтынуодавы Қараванды университеті, Қараванды, Қазақстан; \\ ${ }^{3}$ Қараванды техникалық, университеті, Қараганды, Қазақстан
}

\title{
Гармоникалық интервалдармен байланысты функциялар кластары үйіріндегі Фурье қатарының дербес қосындылары операторының шенелгендігі туралы
}

\begin{abstract}
Мақала гармоникалық интервалдар деп аталатын арнайы жиынтықтар спектрі бар тригонометрикалық полиномдар функцияларын жуықтау теориясының кейбір деректерін зерттеуге арналған. Математикалық модельді зерттеу кезінде құрылғылардың қабылдау ауқымы, адамның сезім мүшелерінің қабылдау ауқымы шектеулі болғандықтан қателік (шу, кедергі, бұрмалау) қабылдау интервалынан тыс болатындай етіп қажетті объектінің жуықтамасын табу көбінесе жеткілікті болады. Гармоникалық интервалдар осындай типтегі мәселелерді белгілі бір деңгейде модельдейді. Мақалада гармоникалық интервалдар деп аталатын арнайы жиынтықтар спектрі бар тригонометрикалық полиномдар функцияларын жуықтау теориясының негізгі компоненттері келтірілген, гармоникалық интервалдар деп аталатын арнайы жиынтықтар спектрі бар тригонометрикалық полиномдар функциясын ең жақсы жуықтау арқылы тригонометрикалық полиномдар функциясын ең жақсы жуықтауды бағалау туралы теоремасы дәлелденді. Гармоникалық интервалдармен байланысты функциялар кластары үйіріндегі Фурье қатарының дербес қосындылары операторының шенелгендігі туралы теоремалар келтірілген, мұндай теорема Лоренц кеңістігі үшін жалпыландырылған және дәлелденген. Негізінен мақала арнайы жиынтықтар спектрі бар тригонометрикалық полиномдар функцияларын жуықтау теориясының практикалық қолдануымен айналысатын ғылыми зерттеушілерге арналған.
\end{abstract}

Kiлm сөздер: гармоникалық интервал, гармоникалық интервалдар спектрі бар тригонометрикалық полиномдар, тригонометрикалық полиномдар функциясын ең жақсы жуықтау, белгіленген функция үшін Фурье қатарының дербес қосындылары операторы, интерполяциялық теорема. 


\author{
Г.А. Есенбаева ${ }^{1}$, Г.А. Есенбаева ${ }^{2}$, А.Т. Касимов ${ }^{3}$, Н.К. Сыздыкова ${ }^{1}$ \\ ${ }^{1}$ Карагандинский университет имени академика Е.А. Букетова, Караганда, Казахстан; \\ ${ }^{2}$ Карагандинский университет Казпотребсоюза, Караганда, Казахстан; \\ ${ }^{3}$ Карагандинский технический университет, Караганда, Казахстан
}

\title{
Об ограниченности оператора частичных сумм ряда Фурье в семействах классов функций, связанных с гармоническими интервалами
}

\begin{abstract}
Статья посвящена исследованию некоторых данных теории приближения функций тригонометрическими полиномами со спектром из специальных множеств, называемых гармоническими интервалами. В силу ограниченности диапазона восприятия приборов, диапазона восприятия органов чувств самого человека при исследовании математической модели часто достаточно найти приближение искомого объекта так, чтобы погрешность (шумы, помехи, искажения) оказалась вне интервала восприятия. Гармонические интервалы в некоторой степени моделируют задачи такого рода. В статье представлены основные компоненты теории приближения функций тригонометрическими полиномами со спектром из гармонических интервалов, доказана теорема об оценке наилучшего приближения функции тригонометрическими полиномами через наилучшие приближения функции тригонометрическими полиномами со спектром из гармонических интервалов. Приведены теоремы об ограниченности оператора частичных сумм ряда Фурье в семействах классов функций, связанных с гармоническими интервалами, обобщена и доказана такая теорема для пространства Лоренца. Статья ориентирована, в основном, на научных исследователей, занимающихся практическими приложениями теории приближений функций тригонометрическими полиномами со спектром из специальных множеств.
\end{abstract}

Ключевые слова: гармонический интервал, тригонометрические полиномы со спектром из гармонических интервалов, наилучшее приближение функции тригонометрическими полиномами, оператор частичных сумм ряда Фурье для заданной функции, интерполяционная теорема.

\section{References}

1 Temlyakov, V.N. (2021). Diskretizatsiia integralnykh norm polinomov s garmonikami iz giperbolicheskogo kresta po znacheniiam v tochkakh [Discretization of integral norms for polynomials with harmonics from a hyperbolic cross by values at points]. Funktsionalnye prostranstva, teoriia priblizhenii $i$ smezhnye voprosy analiza: sbornik statei $k$ 115-letiiu so dnia rozhdeniia akademika Sergeia Mikhailovicha Nikolskogo. Trudy MIAN - Functional spaces, approximation theory, and related issues of analysis. Collection of articles dedicated to the 115th anniversary of the birth of academician Sergei Mikhailovich Nikolsky. Works of MIAS, 312, 282-293 [in Russian].

2 Telyakovsky, S.A. (1983). Ocherki razvitiia matematiki v SSSR [Essays on the development of mathematics in the USSR]. Kiev: Naukova dumka [in Russian].

3 Nursultanov, E.D. (1999). Setevye prostranstva i ikh prilozheniia k zadacham garmonicheskogo analiza [Network spaces and their applications to problems of harmonic analysis]. Doctor's thesis. Moscow [in Russian].

4 Nursultanov, E.D. (1998). Setevye prostranstva i neravenstva Hardi-Littlvuda [Network spaces and HardyLittlewood inequalities]. Matematicheskii sbornik - Mathematical collection, 189, 3, 83-102 [in Russian].

5 Nursultanov, E.D. (1998). Setevye prostranstva i preobrazovanie Fure [Network spaces and the Fourier transform]. Doklad RAN (361) - RAS report (361), 5, 597-599 [in Russian].

6 Berg, J., \& Lefstrom, J. (1980). Interpoliatsionnye prostranstva. Vvedenie [Interpolation spaces. Introduction]. Moscow: Mir [in Russian].

7 Krein, S.G., Petunin, Yu I., \& Semenov, E.M. (1978). Interpoliatsiia lineinykh operatorov [Interpolation of linear operators]. Moscow: Nauka [in Russian].

8 Brudny, Yu.A., Krein, S.G., \& Semenov, E.M. (1986). Interpoliatsiia lineinykh operatorov [Interpolation of linear operators]. Itogi nauki i tekhniki. Seriia Matematicheskii analiz - Results of Science and Technology. Ser. Math. anal., 24, 3-163 [in Russian]. 
9 Tribel, Kh. (1980). Teoriia interpoliatsii, funktsionalnye prostranstva, differensialnye operatory [Interpolation theory, function spaces, differential operators]. Moscow: Mir [in Russian].

10 Tribel, Kh. (1986). Teoriia funktsionalnykh prostranstv [Theory of functional spaces]. Moscow: Mir [in Russian].

11 Yessenbayeva, G.A. (2008). O svoistvakh chastichnykh summ po garmonicheskim intervalam i klassakh funktsii, sviazannykh s nailuchshimi priblizheniiami po garmonicheskim intervalam [On the properties of partial sums over harmonic intervals and classes of functions associated with the best approximations over harmonic intervals]. Vestnik Karagandinskogo universiteta. Seriia Matematika - Bulletin of the Karaganda University. Mathematics Series, 1(49), 29-36 [in Russian].

12 Yessenbayeva, G.A., \& Nursultanov, E.D. (2004). Interpoliatsiia nekotorykh neravenstv i approksimatsiia funktsii trigonometricheskimi polinomami so spektrom iz garmonicheskikh intervalov [Interpolation of some inequalities and approximation of functions by trigonometric polynomials with spectrum from harmonic intervals]. Evraziiskii matematicheskii zhurnal - Eurasian mathematical journal, 1, 3-20 [in Russian].

13 Smailov, E.S., Akishev, G.A., \& Esmaganbetov, M.G. (1986). Teoriia priblizheniia i vlozheniia klassov funktsii mnogikh peremennykh /Approximation theory and embeddings of classes of functions of many variables]. Karaganda: Izdatelstvo Karagandinskogo universiteta [in Russian].

14 Nikolsky, S.M. (1977). Priblizhenie funktsii mnogikh peremennykh i teoremy vlozheniia /Approximation of functions of many variables and embedding theorems]. Moscow: Nauka [in Russian].

15 Bari, N.K. (1961). Trigonometricheskie riady [Trigonometric series]. Moscow: Fizmatlit [in Russian]. 